\section{IDH1: function follows form}

\section{By Kai-Jye Lou, Staff Writer}

In brain cancer, isocitrate dehydrogenase 1 mutations associated with the disease have long puzzled researchers because it was unclear whether the mutations caused a loss of the enzyme's native activity or triggered the gain of an altogether new function. Now, Agios Pharmaceuticals has shown that both scenarios are plausible: the biotech found that cancer-associated mutations in the enzyme give it a new catalytic activity while also stripping it of its native function. ${ }^{1}$

Although it's still unclear whether trying to restore the enzyme's native activity or inhibiting its alternative function will provide a therapeutic benefit in brain cancer, Agios is now seeking to determine the role of isocitrate dehydrogenase 1 (IDH1) in glioma pathogenesis.

The company suspects that inhibition of the mutated enzyme could be an attractive strategy for targeting these tumors and has a program to discover inhibitors against mutant IDH1.

Wild-type IDH1 catalyzes the conversion of isocitrate into $\alpha$-ketoglutarate, which is a key metabolic step in the citric acid cycle, a central component of glucose metabolism.

Mutations at arginine 132 in cytosolic IDH1 are found in about $80 \%$ of grade II-III gliomas and secondary glioblastomas. In February, researchers at Duke University School of Medicine and The Johns Hopkins University published in The New England Journal of Medicine that these mutations in IDH1 could cause the enzyme to gain an unknown function. ${ }^{2}$ Another study by U.S. and Chinese researchers, published in April in Science, suggested that mutational inactivation of IDH1 contributes to tumorigenesis through induction of the hypoxia-inducible factor 1 (HIF1) pathway. ${ }^{3}$

"Since their discovery, the function of IDH mutations in cancer has puzzled cancer geneticists," said Hai Yan, a corresponding author on the NEJM paper and an associate professor in the Department of Pathology at Duke.

In Agios' study, the team first used mass spectrometry to identify changes in glucose metabolites by comparing wild-type cells with cells containing a mutated form of IDH1. Only one metabolite was significantly more abundant in the mutated cells: $R(-)$-2-hydroxyglutarate (2HG) $(p<0.001)$. This metabolite had been previously linked to the development of brain cancer. ${ }^{4}$
The team then used an enzyme activity assay to show that mutant IDH1 indeed gains the ability to catalyze the conversion of $\alpha$-ketoglutarate into $2 \mathrm{HG}$. Other experiments also showed that the enzyme's native function had been disrupted by the mutations.

The results were published in Nature.

"The Agios team demonstrated that a single amino acid substitution in the IDH1 active site allows the enzyme to acquire an entirely new activity to produce $2 \mathrm{HG}$," said interim CSO Shin-San Michael Su, who was corresponding author on the Nature paper. "Analysis of tumor samples of brain cancer patients with the IDH1 mutation revealed up to 100-fold elevations in concentrations of $2 \mathrm{HG}$."

"The biochemical analysis seems quite convincing that mutated IDH1 not only loses its normal catalytic activity in producing $\alpha$-ketoglutarate but also gains a new catalytic function," said Yue Xiong, a corresponding author on the April Science paper and a professor of biochemistry and biophysics at the Lineberger Comprehensive Cancer Center at The University of North Carolina at Chapel Hill School of Medicine.

Agios also reported X-ray crystal structure data suggesting that the mutations at arginine 132 result in the formation of a distinct active site on IDH1. The company team said this change could be responsible for the mutant enzyme's altered function.

"Now that we believe the mutations are adding a new function to the enzyme, one could imagine designing a small molecule that could fit into the mutant IDH1 active site and knock out its function," said Donald Parsons, the other corresponding author on the February NEJM paper and an assistant professor in the Department of Pediatrics at the Baylor College of Medicine. "This work gives hope that a hypothetical small molecule inhibitor of mutant IDH1 could be used to treat a large subset of low-grade and early gliomas and secondary glioblastoma."

\section{IDH1's unanswered questions}

"The findings create opportunities for therapeutic intervention in brain cancer, and other cancers where IDH1 mutations are present, by using new drugs that can target the IDH1 metabolic pathway," Su told SciBX. "We believe that our IDH1 program has the potential to address the needs of patients with diseases such as gliomas, and we are prioritizing the program to expedite its development. Before this research, IDH1 was just one of multiple potential targets in our ongoing cancer metabolism research. After these findings, it has become one of our top-priority programs for drug discovery."

Yan cautioned, however, that "there is still a ways to go before we will know whether we can use IDH1 as a therapeutic target. We would need to know if this unique change in IDH1 is targetable. Future work should focus on how this gain of function causes tumors and then progress to studying how to target these pathways. The functional significance of isocitrate dehydrogenase mutations in human cancer remains, to a large extent, a mystery." 
Yan also thinks additional work should focus on the association between mutant IDH1 and 2HG. "The other key question is to figure out if and why $2 \mathrm{HG}$ is needed by the cancer cells. It will be important to understand how the $2 \mathrm{HG}$ metabolite affects cell behavior, because if it turns out to be the fuel that these cells need, researchers can develop ways to block its activity," he told SciBX.

Xiong said it "will be very interesting to determine the structural basis of the newly gained activity in mutant IDH1," as such information could aid the development of compounds that specifically target the mutant form of the enzyme over the wild-type form.

Agios is now conducting validation experiments both in-house and with academic collaborators.

"Validation experiments will demonstrate whether the IDH1 mutation is an initiation event in the development of cancer or whether it is required for ongoing tumor maintenance," said Su. "These experiments will demonstrate how best to target IDH1 in cancer."

Agios also is exploring the potential of $2 \mathrm{HG}$ as a biomarker. According to $\mathrm{Su}, 2 \mathrm{HG}$ is found in high concentrations in tumor samples in conjunction with the IDH1 mutation. "It may well be possible, depending on the tumor type, for sufficient levels to be present in the blood or other body fluids for a simple, noninvasive diagnostic test to be developed, which would enable rapid diagnosis and earlier treatment," he said.

Agios said it holds the IP around mutant IDH1 as a potential target and $2 \mathrm{HG}$ as a potential biomarker. The mutant IDH1 program is not currently licensed.

Lou, K.-J. SciBX 2(48); doi:10.1038/scibx.2009.1749

Published online Dec. 17, 2009

REFERENCES

1. Dang, L. et al. Nature; published online Nov. 22, 2009; doi:10.1038/nature08617

Contact: Shin-San Michael Su, Agios Pharmaceuticals, Boston, Mass.

e-mail: michael.su@agios.com

2. Yan, H. et al. N. Engl. J. Med. 360, 765-773 (2009)

3. Zhao, S. et al. Science 324, 261-265 (2009)

4. Aghili, M. et al. J. Neurooncol. 91, 233-236 (2009)

COMPANIES AND INSTITUTIONS MENTIONED

Agios Pharmaceuticals, Boston, Mass.

Baylor College of Medicine, Houston, Texas

Duke University School of Medicine, Durham, N.C.

The Johns Hopkins University, Baltimore, Md.

The University of North Carolina at Chapel Hill School of Medicine, Chapel Hill, N.C. 